\title{
Invariants for quantum supergroups and some applications
}

\author{
JON ROBERT LINKS
}

The study of Hopf algebras is not a new one in mathematics although in recent years it is an area which has gained increased attention. This situation has arisen since the advent of quantum groups (defomations of Lie algebras) which were introduced as a means of solving the Yang-Baxter equation. It was quickly realised that quantum groups had the structure of a Hopf algebra and that it was such a structure that was fundamental in solving the Yang-Baxter equation. It has also been realised that a $\mathbb{Z}_{2}$-graded Hopf algebra (or Hopf superalgebra) plays a similar role in solving the YangBaxter equation with quantum supergrops (deformations of Lie superalgebras) being an example of such an algebra.

The work of this thesis is concerned with developing techniques of constructing invariants for abstract Hopf superalgebras and investigating their applications with respect to quantum supergroups. Different methods are considered for constructing invariants in Chapter 2 enabling analogues of the Gel'fand invariants to be obtained for quantum groups.

The study of invariants has been central to the development of the representation theory of Lie algebras and it is natural to expect that there will be a similar importance for quantum groups. This is shown to be so for the quantum group $U_{q}(g l(n))$ in Chapter 3 . The techniques developed in Chapter 2 are applied to construct raising and lowering operators for $U_{q}(g l(n))$ and also to present an evaluation of the matrix elements of the generators in agreement with previously known results.

The relationship between the Yang-Baxter equation and knot theory is well known and a major part of the thesis is devoted to investigating the role of quantum supergroups in this respect. Exploiting the fact that the braid generator arising from the representation of a quantum supergroup is itself an invariant enables a Markov trace to be defined which in turn permits link polynomials to be constructed. The use of representations of quantum supergroups allows results to be obtained which are not available from other methods. Particularly, consideration is given to yielding new two variable link polynomials.

Received 11th January, 1994.

Thesis submitted to the University of Queensland June, 1993. Degree approved November 1993. Supervisor: Dr Mark D. Gould.

Copyright Clearance Centre, Inc. Serial-fee code: 0004-9729/94 \$A2.00+0.00. 
Finally, the problem of Baxterisation to obtain a full solution to the Yang-Baxter equation is considered. It is demonstrated how this problem may be simplified in the framework of invariant operators and how the use of representations of quantum supergroups can allow two parameter solutions to be obtained.

Department of Mathematics

The University of Queensland

Queensland 4072

Australia 